\title{
An efficient composite membrane to improve the performance of PEM reversible fuel cells
}

\author{
Gabriele G. Gagliardi ${ }^{I}$, Carlotta Cosentini ${ }^{l}$ and Domenico Borello ${ }^{l}$ \\ ${ }^{1}$ Department of Mechanical and Aerospace Engineering, University of Rome La Sapienza, Rome, Italy
}

\begin{abstract}
The aim of this study is to develop composite Nafion/GO membranes, varying GO loading, to be used in a Unitized reversible fuel cell comparing its performance with the baseline Nafion. Water uptake, ion exchange capacity (IEC), tensile strength, and SEM (scanning electron microscope) analysis are discussed. The SEM analysis revealed how the GO is homogeneously disposed into the Nafion matrix. The addition of GO improves the membrane tensile strength while reducing the elongation ratio. Water uptake, IEC enhance with the increasing of GO content. Regarding fuel cell mode, the performance is analysed using a polarization curve on a MEA with an effective area of $9 \mathrm{~cm}^{2}$. The composite membrane demonstrated higher mechanical strength, enhanced water uptake so higher performance in fuel cell mode. Despite the power absorbed from the electrolysis is higher when using a composite membrane, the beneficial effect in FC mode resulted in a slightly higher round trip efficiency. The GO-Nafion membrane was not able to maintain its performance with increasing the operating time, so potentially leading to a lower lifetime than the Nafion bare.
\end{abstract}

\section{Introduction}

Considering the global warming, new technologies must be efficient and especially respectful of the environment. Indeed, the emissions of greenhouse gas, like carbon dioxide, carbon monoxide or other like SOx and NOx, must be avoided at all costs. The energy also must be produced without fossil fuel because the latter's reserves diminish and come dangerously to their ends. Then, renewable energies are worthwhile because of the tremendous and limitless amount of energy that can be produced. New technologies like electrolysers (and fuel cells), can be linked to renewable sources, which have non-continuous production [1] to store the surplus of energy in the form of gas that can be used in a second time with fuel cells. Among different types of fuel cells, the Unitized Reversible Fuel Cells (RFCs) are an innovative and promising technology able to accomplish two different tasks: power production (fuel cell mode) and energy storage (electrolyser mode) in one device [2]. In addition to the versatility and the reversibility, this technology is characterized by several advantages such as low operating temperature, fast start ups, sustainable operation at a high current density, low volume, low weight of the stack and suitability for discontinuous operation. However, some technical challenges are still to be overcome. The basic challenges are related to the materials cost and to the durability. To date, the operational lifetime for real life application does not meet the requirements for the art technologies state, such as $40,000 \mathrm{~h}$ for stationary applications, 20,000 h and $5000 \mathrm{~h}$ for mobile applications [3]. Despite many efforts to develop the URFC-PEM fuel cell technology in past decades, its major barriers including durability and cost for commercialization purpose have not been solved. To overcome those issues, research has investigated ways of modifying the Nafion matrix for many reasons: (a) compared to other fuel cell components, the production cost of Nafion is significantly higher; (b) the durability is not efficient for long cycle unit cell operation due to the swelling nature of the membrane; and (c) fuel permeability. Although many investigations have been undertaken, up to now, not many membrane-based studies have been reported for URFC applications. A low-cost, highly stable advanced composite-structured membrane should be proposed to replace the more expensive Nafion membranes. Sulfonated polyether ether ketone (sPEEK) and sulfonated polyphenylene oxide (SPPO) can be considered as efficient organic compounds instead of conventional Nafion due to their low cost, availability, and easy sulfonation processes. The sulfonated polymer membrane can increase the proton conductivity, flexibility, and stability of the membrane. To augment the advanced properties in the membrane, functionalized inorganic materials must be dispersed in the polymer membrane matrix. The inorganic materials can enhance the mechanical and thermal stability of the polymer membrane due to strong interfacial interaction between the polymer main chain and inorganic functional properties. Furthermore, the incorporation of inorganic material can act as a barrier for the fuel crossover in the membrane [4]. Graphene Oxide (GO) is a highly hydrophilic material and exhibits a reasonable level of proton conductivity. It was reported

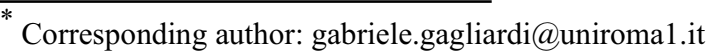


that the incorporation of GO into the Nafion electrolyte increases the mechanical strength and gas impermeability of the membrane so enhancing the performance in fuel cell mode giving the opportunity to reduce the thickness of the membrane so reducing the total cost [5]. The work reported here is aimed to improve the performance and the durability of Nafion membranes for URFC application. Therefore, Nafion and GO-Nafion composite membranes with variable GO loading were fabricated, characterised, and tested in-situ.

\section{Materials and method}

\subsection{Materials}

Materials and precursor were obtained from several companies: GO sheets and hydrogen peroxide (34\%) from Sigma Aldrich; sulfuric acid (98\%) from Alfa Aesar; Nafion dispersion, GDL and catalysts, PtC at the anode and the cathode, from Fuel cell store.

\subsection{Preparation of membranes}

Nafion and composite Nafion-GO membranes were prepared using casting method, the process of formation of film or membrane on a flat surface by the evaporation of the solvent [6]. Regarding Nafion-GO membranes, three different loading were considered: $0.5,1$ and $1.5 \%$. For membrane preparation the following procedure was adopted: a) pour Nafion solution in a flat petri dish; b) dry for 2 hours at $100^{\circ} \mathrm{C}$; c) dry it at $120^{\circ} \mathrm{C}$ for 1 hour in the oven to anneal. Afterwards, the membranes were treated at $80{ }^{\circ} \mathrm{C}$ by immersion in the following sequence (each procedure lasted $1 \mathrm{~h}$ ): in water, in $3 \% \mathrm{H}_{2} \mathrm{O}_{2}$, in water, in $0.5 \mathrm{M} \mathrm{H}_{2} \mathrm{SO}_{4}$ and in water. Then, the membranes were immersed in water overnight.

\subsection{Characterization}

The main properties, such as water uptake (WU), ion exchange capacity (IEC), microscopic structure and tensile strength, were evaluated. WU and IEC are relevant tests to characterize a membrane as proton exchange membrane due to the influence of water on the proton transport. Microscopic structure and tensile strength measurement were carried out to understand the dispersion of GO in the Nafion matrix and the improvement of mechanical properties. For a better comprehension, all the data are compared with that of the commercial membrane and a casted Nafion.

\subsubsection{Water uptake}

Water uptake (WU) was determined gravimetrically by recording the wet and dry mass of the membranes using the following steps (repeated three times): the membranes were placed in water at room temperature for $24 \mathrm{~h}$, and then their wet weight was measured. To measure the wet weight, the water on the surface was absorbed using dry filter paper. After this, the wet membrane samples were dried at $100{ }^{\circ} \mathrm{C}$ (until no more 75 weight variation was detected) and their dry weight was recorded. The water uptake (WU) was measured using the Equation 1, where $\mathrm{WE}_{\mathrm{w}}$ and $\mathrm{WE}_{\mathrm{d}}$ denote the wet weight and the dry weight, respectively.

$$
W \mathrm{U}=\left(W E_{\mathrm{w}}-W E_{\mathrm{d}}\right) / W E_{\mathrm{d}}
$$

\subsubsection{Ion exchange capacity}

The ion exchange capacity (IEC), expressed as milliequivalent of ion exchange groups per gram of the membrane $(\mathrm{meq} / \mathrm{g})$, is an important parameter because the ionic transport properties depend on the amount of the ion exchange groups. The IEC and WU are usually correlated: an increase of IEC induces high water content, but the mechanical strength of the membrane drops [7]. Membranes Ion Exchange Capacity was evaluated by acid-base titration method. The desired membrane was soaked in $0.1 \mathrm{M} \mathrm{HCl}$ for $24 \mathrm{~h}$. After thoroughly rinsing the membrane with water, it was immersed in saturated $\mathrm{NaCl}$ for $72 \mathrm{~h}$ to exchange the $\mathrm{H}^{+}$ ions for $\mathrm{Na}^{+}$ions. Then, the proton release was evaluated by titrating the solution with $0.01 \mathrm{M} \mathrm{NaOH}$ at room temperature with phenolphthalein as indicator. The IEC was obtained by using the Equation 2:

$$
I E C=\left(V O L_{\mathrm{NaOH}} x M_{\mathrm{NaOH}}\right) / W E_{\mathrm{d}}
$$

\subsubsection{Scanning electron microscope}

Scanning electron microscope (SEM) was used to record surface morphology information of the sample by recording secondary electrons emitted from the material due to inelastic scattering [8]. Using this method, the primary electron beam is scanned across the sample, exciting the electrons on the surface, which are then detected to produce 3D images. SEM can successfully be used to investigate particle distribution throughout the thickness of the membranes at both nanometre and micrometre scale. The analysis is carried out through Electron microscopy analyzer using a Zeiss EM10 SEM, in the following operating conditions: $20 \mathrm{kV}$ acceleration voltage, beam current between 6 and $14 \mathrm{pA}$ and BSD detector with four elements, gain +3 . By analysing the cross sections of the composite membranes by SEM, the distribution of the filler particles could be evaluated.

\subsubsection{Tensile strength}

Tensile strength and elongation are both important mechanical properties regarding polymeric membrane because they indicate the maximum stress to which a material can resist before breaking. This test was performed using a Zwick/Roel Z010 following the standard method D882-02. 5 samples were cut in strips of uniform width, $11 \mathrm{~mm}$, placed in the grips of the machine and tested at a strain rate of $1 \mathrm{~mm} / \mathrm{min}$. 


\subsection{Fuel cell tests}

The MEA was obtained by hot pressing the membrane sandwiched between the commercial electrodes purchased from Fuelcellstore. The electrochemical performance (cell voltage and electrical current) of the fuel cell were measured by using a test station consisting of an Agilent E3631A DC power supply to perform the electrolysis and the TTi LD300 Electronic DC Load to carry out the polarization curve in the fuel cell mode. The power density was calculated as the product of cell voltage and current density. The power density was plotted against the current density (P-I curve) to determine the peak power density (Pmax) at each test.

\section{Results and discussion}

\subsection{Membrane characterization}

SEM images of the GO-Nafion composite membranes with different loading $(0.5,1$ and $1.5 \%)$ are presented in Fig. 1. The composite membrane showed the GO sheets dispersed horizontally in the polymer layer and perpendicular to the direction of reactant supply to electrodes.
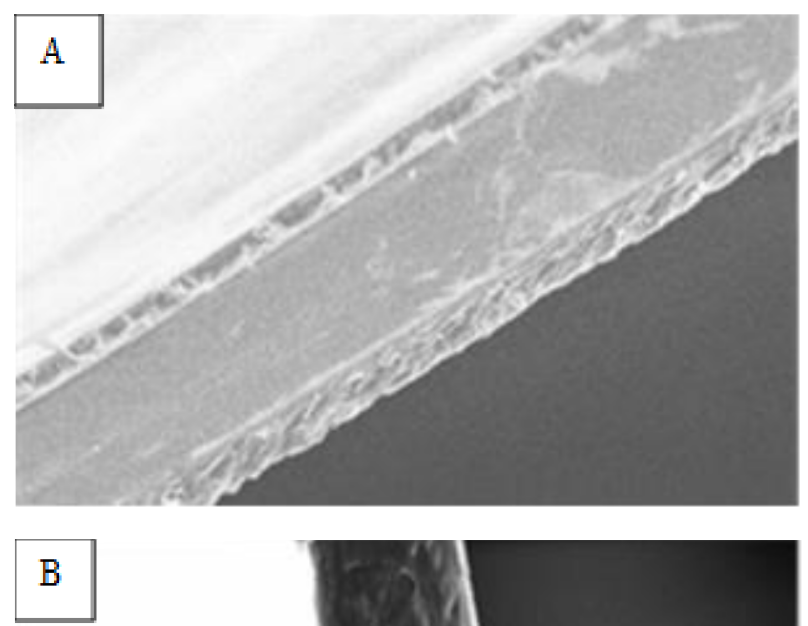

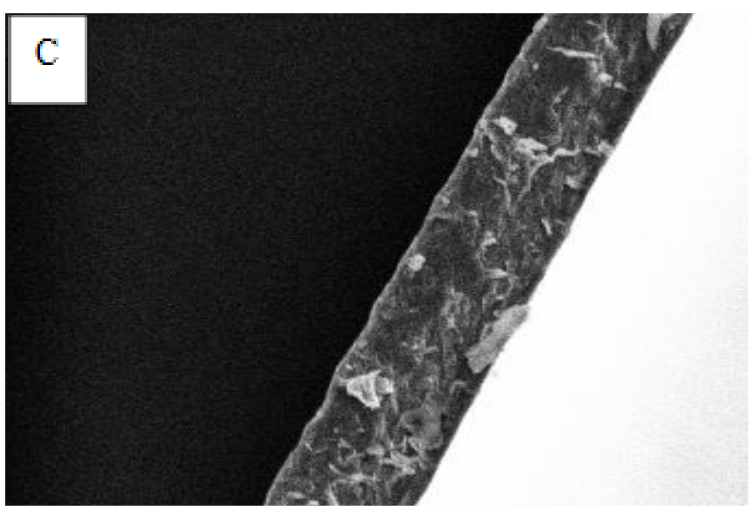

Fig. 1. SEM images for recast and GO membranes. A for $0.5 \%$, $\mathrm{B}$ for $1 \%$ and $\mathrm{Cf}$ or $1.5 \%$.

Table 1 displays the membrane characteristics of water uptake, swelling, and IEC. For all loading, the presence of GO in the polymer structure has led to an increase in swelling and has improved water uptake due to the hydrophilic nature of graphene oxide.

Table 1. Water uptake, swelling, and ion exchange capacity of the fabricated membranes.

\begin{tabular}{|c|c|c|c|}
\hline Membrane & $\begin{array}{c}\text { Water } \\
\text { uptake \% }\end{array}$ & $\begin{array}{c}\text { Swelling } \\
\text { ratio (\%) }\end{array}$ & $\begin{array}{c}\text { IEC } \\
\text { (meq/g) }\end{array}$ \\
\hline Nafion & 16.55 & 24.07 & 0.80 \\
\hline $\begin{array}{c}\text { Nafion-GO } \\
(0.5 \%)\end{array}$ & 28.10 & 25.37 & 0.87 \\
\hline $\begin{array}{c}\text { Nafion-GO } \\
(1 \%)\end{array}$ & 31.16 & 27.38 & 0.89 \\
\hline $\begin{array}{c}\text { Nafion-GO } \\
(1.5 \%)\end{array}$ & 31.88 & 29.63 & 0.91 \\
\hline
\end{tabular}

The beneficial effect of GO on the IEC is controversial. Some authors [5] claimed that the IEC decreased with the addition of GO, when compared to the recast Nafion, due to the less Nafion amount in the GO membranes and a reduction in ionic channels. However, authors [9] claimed that $\mathrm{s}$ non-uniform dispersion with agglomerates could potentially have a higher IEC as less ionic channels are obstructed by the graphene oxide. The tensile strength and elongation ratio data of the recast Nafion and composite membrane were summarized in Table 2.

Table 2. Tensile strength and elongation ratio of the fabricated membranes.

\begin{tabular}{|c|c|c|}
\hline Membrane & $\begin{array}{c}\text { Tensile strength } \\
{[\mathbf{M P a}]}\end{array}$ & $\begin{array}{c}\text { Elongation ratio } \\
(\mathbf{\%})\end{array}$ \\
\hline Nafion & 16.55 & 75 \\
\hline $\begin{array}{c}\text { Nafion-GO } \\
(0.5 \%)\end{array}$ & 28.10 & 65 \\
\hline $\begin{array}{c}\text { Nafion-GO } \\
(1 \%)\end{array}$ & 31.16 & 50 \\
\hline
\end{tabular}




\begin{tabular}{|c|c|c|}
\hline $\begin{array}{c}\text { Nafion-GO } \\
(1.5 \%)\end{array}$ & 31.88 & 40 \\
\hline
\end{tabular}

All GO/Nafion membrane showed a tensile strength higher than the bare Nafion membrane due to the inclusion of GO into the perfluorosulfonic matrix. The more GO is included in the membrane, the higher tensile strength is obtained, as stated by several authors [10-11]. However, as can be observed, the elasticity of the GO membrane is very low compared to Nafion. This result is in agreement with literature. In fact, Bayer et al. [11] claimed that the elongation of the GO membrane before rupture is only $22 \pm 1 \%$, compared with $411 \pm 14 \%$ in Nafion, so the introduction of GO results in a decrease in elongation. To obtain good performances, membrane should have high elongation ratio and high tensile strength. However, high tensile strength is often chosen over the elongation ratio. A good compromise between a gain obtained from the water uptake, IEC, and tensile strength and the loss of the elongation rate, is obtained by using the membranes with 1 and $1.5 \%$ of GO loading, which represent the optimum range. In this work, MEAs were fabricated using GO membranes with $1 \%$ of loading, a compromise solution between the enhancement obtained and material quantity, so costs.

\subsection{In-situ tests}

The current-voltage (I-V) characteristics curves for the prepared GO and Nafion casted composite membrane are studied in both electrolysis and fuel cell mode. Single cell water electrolysis polarization curves were depicted in Figure 2.

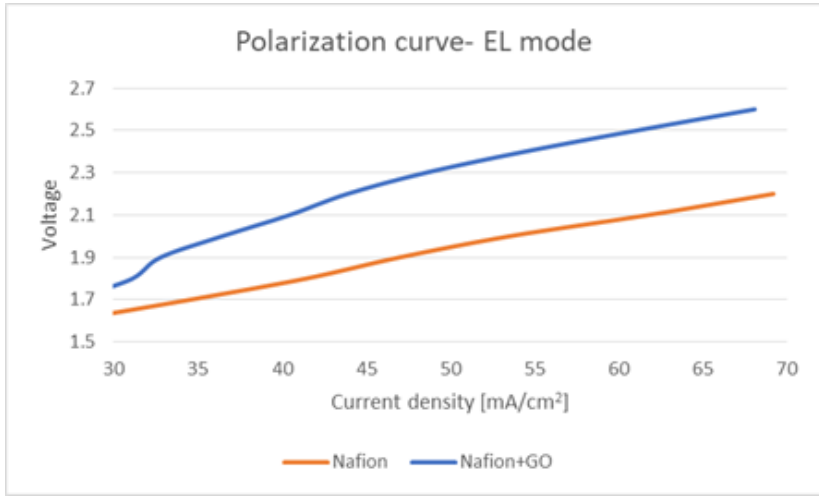

Fig. 2. Water electrolysis polarization curves

The current onset in the activation region is slightly favourable for the cell with plain Nafion, whereas at higher current densities, higher voltages are required for the cell assembled with the GO composite membrane. To the best of our knowledge, there are no studies in the literature on the use of graphene oxide as a polymer electrolyte in water electrolysis. Therefore, this phenomenon needs to be better elucidated, carrying out tests at different temperature and different GO loading, because on a first attempt the analysed behaviour could be due to the presence of the functionalized inorganic filler dispersed in the polymer matrix, which lowers the proton conductivity of the membrane, phenomenon emphasized at low temperature. From the polarization curves obtained in the fuel cell mode, the trend observed is the opposite (Figure 3).

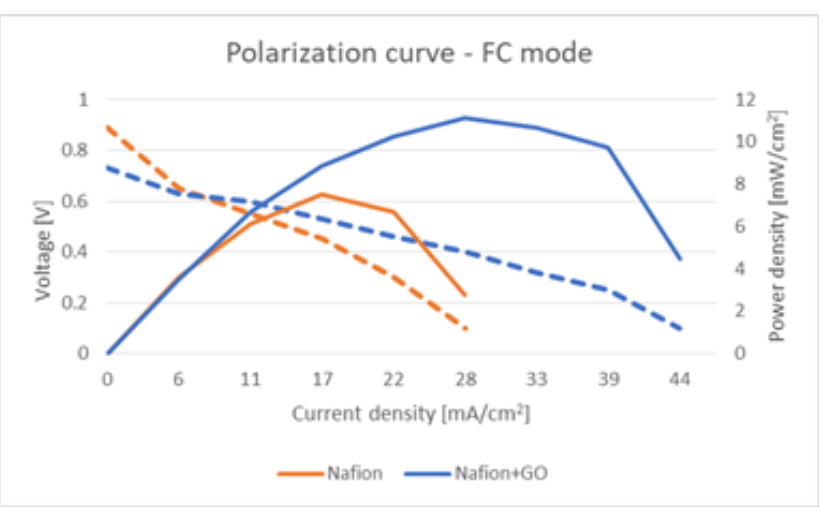

Fig. 3. Fuel cell polarization curves

The composite GO membrane showed higher performance than that of recast Nafion. The maximum power density of the composite membrane and the operating range were $48 \%$ and $57 \%$ more than those recorded for the bare Nafion. This was because the graphene oxide retained more water and enhance the ion exchange capacity, so those beneficial effects mitigate the drop in the proton conductivity [12]. Figure 4 shows the maximum FC generated power density for more than 15 cycles to investigate the energy performance drop of the device when it performs numerous continuous cycles.

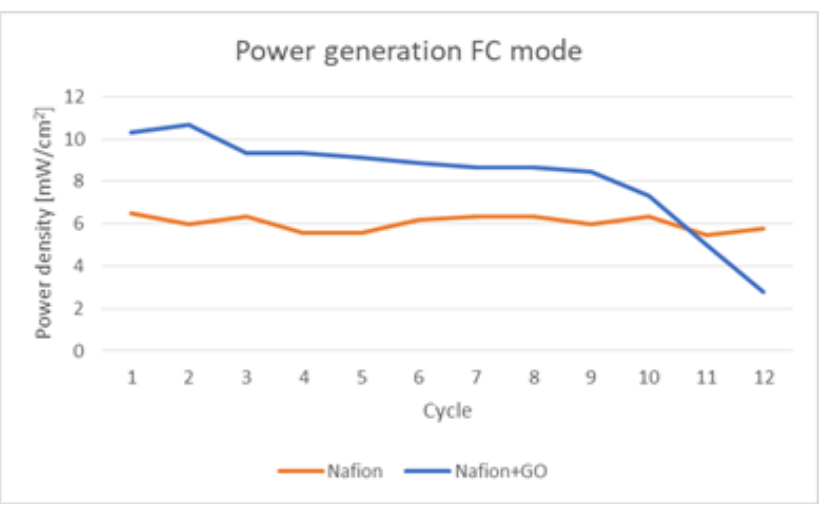

Fig. 4. Maximum power output over cycles

As is clear from the above figure, the maximum power density delivered by the fuel cell assembled with the GO membrane was initially higher than that of the bare Nafion. However, the performance started to drop after the tenth cycle reaching a maximum value of $8 \mathrm{~mW} / \mathrm{cm}^{2}$ then collapsing up to $3 \mathrm{~mW} / \mathrm{cm}^{2}$ at the twelfth cycle. This trend was expected due to electrochemical carbon corrosion at high voltage, one of the critical determinants of the lifetime of polymer electrolyte membrane fuel cells [13]. Considering the results obtained in both WE and FC, the round-trip efficiency (RT) for the Nafion membrane was $21 \%$, slightly lower than the RT efficiency of the GO composite membrane that reached a value of $23 \%$. Despite the small improvement, the RT is 
still far from being an acceptable value for URFC commercialisation and global uses besides the fact that is should be kept constant for hundreds of hours. However, this was a first study performed on the application of GO membranes for URFC application that requires further investigation.

\section{Conclusion}

In this study, Nafion and GO-Nafion membranes were fabricated via solution casting to be tested in a PEM reversible fuel cell. The membranes were characterised, and it was confirmed SEM that graphene oxide was successfully incorporated into the Nafion structure. The composite membranes exhibited better tensile strength and higher swelling and water uptake, but with lower tensile strength. In-situ testing revealed that the composite membrane generally had higher FC performance, leading to an initial slight improvement in term of efficiency, even if with a lower durability due to the carbon corrosion that occurs during the WE mode. It is important to note that the development of GO-Nafion composite membranes for URFC is not present in literature so the behaviour of the composite membranes presented here should be deepen in term of different operating conditions and different filler loading.

\section{References}

[1] T. Sadhasivam, K. Dhanabalan , S. Roh, T. Kim et al.. International Journal of Hydrogen Energy, 42 (2017)

[2] P. Biddyut, J. Andrews. Renewable and Sustainable Energy Reviews 79, (2017)

[3] G. Mohamed, et al. International journal of hydrogen energy 39, (2014)

[4] H. Junoh et al. Membranes 10, (2020)

[5] A. Ibrahim et al.. International Journal of Hydrogen Energy, 45 (2020)

[6] Jung, H.-Y.; Cho, K.-Y.; Lee, Y.M.; Park, J.-K.; Choi J.-H.; Sung Y.-E. Journal of Power Sources 163, (2007)

[7] Yasukawa, M.; Suzuki, T.; Higa, M. MembraneBased Salinity Gradient Processes for Water Treatment and Power Generation $1^{\text {st }}$ edition (2018)

[8] Leng, Y.; Materials Characterization. 2008, Singapore:John Wiley \& Sons (Asia) Pte Ltd.

[9] Kumar, R.; Xu, C.; Scott, K. RSC Adv, (2012).

[10] Wang, L.; Kang, J.; Nam, J.; Suhr, J.; Prasad, A.; Advani, S. ECS Electrochemistry Letters 4, (2015).

[11] Bayer, T.; Bishop, S.R.; Nishihara, M.; Sasaki, K.; Lyth, S.M. Journal of power sources 272, (2014)

[12] Kumar, R.; Xu, C.; Scott, K. RSC Adv. 2, (2012)

[13] S. Jang, H.Kim. J. Am. Chem. Soc. 132, (2010) 